\title{
High-Resolution Search for Pentaquark Partners in Hall $A$ at Jefferson Lab
}

\author{
J.-O. Hansen, for the Jefferson Lab Hall A Collaboration
}

Jefferson Lab, Newport News, Virginia 23606, USA

\begin{abstract}
We have carried out a high-resolution search $(\sigma=1.5 \mathrm{MeV})$ for narrow exotic resonances $(\Gamma<10 \mathrm{MeV})$ in the mass range $M \approx 1500-1850 \mathrm{MeV}$ in $e p \rightarrow e^{\prime} K^{+} X, e p \rightarrow e^{\prime} K^{-} X$ and $e p \rightarrow e^{\prime} \pi^{+} X$ electroproduction at forward angles $\left(\theta^{C M} \approx 6-7^{\circ}\right)$. Such narrow resonances would be candidates for partner states of the speculative $\Theta^{+}(1540)$ pentaquark. The experiment employed a $5 \mathrm{GeV} \mathrm{CW}$ electron beam incident on a liquid hydrogen target and two high-resolution magnetic spectrometers covering a total center-of-mass solid angle of $\Delta \Omega \approx 30-40 \mathrm{msr}$. We do not observe a statistically significant signal in any of the three reaction channels. Upper limits on the production cross sections were determined to be between 3 and $16 \mathrm{nb} / \mathrm{sr}$, depending on the channel and the assumed width of the state. In addition, precise electroproduction data of the $\Lambda(1116), \Sigma(1193)$ and $\Lambda(1520)$ resonances were obtained for calibration purposes.
\end{abstract}

Keywords: pentaquarks, exotics, baryon electroproduction, partner states

PACS: $14.80 .-\mathrm{j}, 13.60 . \mathrm{Rj}, 14.20 . J n, 14.20 . \mathrm{Gk}$

\section{INTRODUCTION}

Recent claims of the observation [1] of a narrow exotic $S=+1$ baryonic state, the $\Theta^{+}(1540)$, have generated considerable experimental and theoretical interest. If confirmed, the $\Theta^{+}$could be the lowest-mass member of an antidecuplet of pentaquarks, which has been predicted in the framework of the Chiral Quark Soliton Model [2]. Other identifications are also possible. For example, the narrow width of the $\Theta^{+}$has been explained in terms of isospin-violating strong decays, which has led to the suggestion of an isotensor multiplet of pentaquarks [3].

If the $\Theta^{+}$pentaquark exists then other members of its symmetry group should be observable as well, provided that they are sufficiently narrow. Both the Chiral Quark Soliton Model and the isotensor multiplet model predict relatively narrow $(\Gamma<30-$ $50 \mathrm{MeV}$ ) partner states in the mass region $M \approx 1500-2000 \mathrm{MeV}$.

In this paper, we report the results of a search for the possible $\Sigma_{5}^{0}$ and $N_{5}^{0}$ members of the antidecuplet and for the isotensor partner $\Theta^{++}$. The reactions $e p \rightarrow e^{\prime} K^{+} X$, $e p \rightarrow e^{\prime} \pi^{+} X$ and $e p \rightarrow e^{\prime} K^{-} X$ were investigated for evidence of narrow structures in the reconstructed missing mass $\left(M_{X}\right)$. The measurements covered a limited range of forward scattering angles, which did not allow a partial-wave analysis. However, excellent mass resolution was achieved. Precise, high-statistics data of the known $\Lambda(1116), \Sigma(1193)$ and $\Lambda(1520)$ resonances were obtained for calibration purposes. The results presented here are near final. 


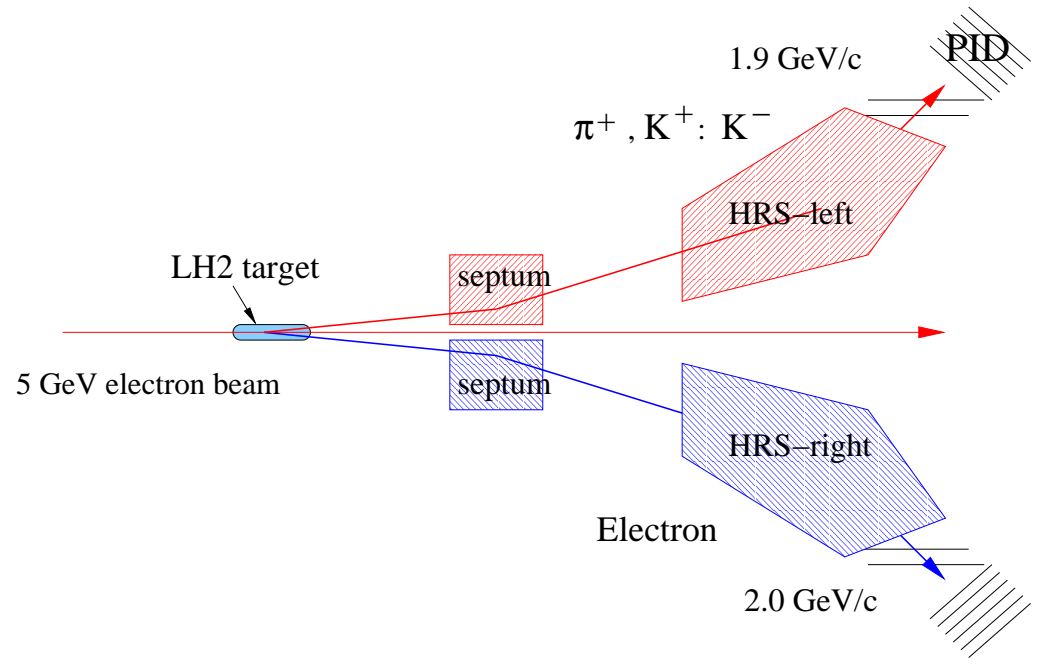

FIGURE 1. Experimental setup.

\section{EXPERIMENT}

The experiment ran in Hall A at Jefferson Lab in May 2004 using a 5 GeV continuouswave electron beam incident on a $15 \mathrm{~cm}$ long liquid hydrogen target. Scattered electrons were detected in one of the Hall A High-Resolution Spectrometers (HRS) in coincidence with electroproduced hadrons that were measured in the second HRS. The experimental setup is illustrated in Figure 1. Both HRS were positioned at $6^{\circ}$ forward angles, using septa on each arm to reach this small scattering angle. Each spectrometer had an effective acceptance of approximately $4 \mathrm{msr}$ in solid angle and $\pm 4.5 \%$ in momentum. For the production runs, to achieve the desired missing mass coverage, the central momentum of the electron HRS was stepped from 1.70 and $2.02 \mathrm{GeV} / \mathrm{c}$, while the central momentum of the hadron HRS was changed between 1.89 and $2.10 \mathrm{GeV} / \mathrm{c}$.

Both spectrometers used a QQDQ magnet arrangement for focusing and a $45^{\circ}$ upward bend. The detector packages, placed behind the magnetic elements, were equipped with four planes of vertical drift chambers for tracking and two planes of fast hodoscopes for triggering. Careful calibration of the trigger system yielded a coincidence timing resolution of $600 \mathrm{ps}$ FWHM. The scattering vertex resolution of each spectrometer was $2.5 \mathrm{~cm}$ FWHM. The electron HRS employed a $\mathrm{CO}_{2}$ gas Cherenkov counter for pion rejection. For the kaon measurements, clean particle identification in the hadron HRS was particularly important because of the very high ratio of $\pi / K$ rates. For this purpose, the hadron HRS was instrumented with two aerogel and a ring-imaging Cherenkov detector as well as a lead glass shower counter. Combined with the coincidence time-offlight measurement, a total pion rejection factor of $3 \times 10^{4}$ was achieved.

The average momentum transfer carried by the virtual photon was $Q^{2} \approx 0.1(\mathrm{GeV} / \mathrm{c})^{2}$. For the kaon (pion) kinematics, the average center-of-mass scattering angle was $\theta_{\gamma^{*} K}^{C M} \approx$ $6^{\circ}\left(\theta_{\gamma^{*} \pi}^{C M} \approx 7^{\circ}\right)$, and the angular acceptance was $\Delta \Omega_{K}^{C M} \approx 40 \operatorname{msr}\left(\Delta \Omega_{\pi}^{C M} \approx 30 \mathrm{msr}\right)$. 


\section{ANALYSIS}

The yields measured in each spectrometer were corrected for detector and trigger efficiencies and dead-time. Cuts on the data from the PID detectors were applied to select appropriate particle types, and vertex and coincidence time cuts were used to reduce background from accidentals. The missing mass, $M_{X}$, was reconstructed using the measured momenta of the electron and kaon (pion). The resulting spectra were corrected for the missing mass acceptance, which varied strongly within the mass range covered by each kinematic setting because of the limited momentum acceptance of the spectrometers. All spectra were normalized by luminosity.

Calibration data were taken in the $e p \rightarrow e^{\prime} \pi^{+} X$ channel in the mass range of the neutron, and in the $e p \rightarrow e^{\prime} K^{+} X$ channel covering the range where the $\Lambda(1116)$ and $\Sigma(1193)$ could be seen. The missing mass resolution was determined to be $3.5 \mathrm{MeV}$ FWHM. Using the well-known masses of the calibration states, the accuracy of our reconstructed missing mass was determined to be better than $3 \mathrm{MeV}$.

In the search regions, the missing mass data from several kinematics (up to 8) were combined to arrive at the final spectra used for the peak search analysis (see below). The individual data sets overlapped to some extent, allowing a check of the corrections and normalizations. After finalizing the detector analysis, all transitions were found to be smooth, requiring no ad-hoc scaling factors. Background was not subtracted from any of the spectra.

To quantify any possible observations (or non-observations), we performed a systematic search for peaks in the final missing mass spectra of the three reaction channels. Within a $50 \mathrm{MeV}$ wide window, we fitted a linear background to the data. Then a BreitWigner peak convoluted with a Gaussian of width $\sigma=1.5 \mathrm{MeV}$ was added to this background at the center of the window, the fit was repeated, and the difference of the $\chi^{2}$ of the two fits, $\Delta \chi_{\text {exp }}^{2}\left(M_{X}\right)$, was recorded. Here, the Gaussian simulated the experimental missing mass resolution, and widths of $\Gamma=1,3,5$, and $8 \mathrm{MeV}$ were assumed for the Breit-Wigner function. This procedure was repeated after shifting the window across the measured missing mass range in steps of $2 \mathrm{MeV}$. For the $\Theta^{++}$search, $\chi^{2}$ was replaced with the log-likelihood because of the low statistics of the data. A strong improvement of $\chi^{2}$ (or log-likelihood, respectively) indicated a candidate for a signal. The statistical significance of the most prominent peak candidates was determined using the standard method of computing the Poisson probability for the background (estimated from sidebands) to fluctuate to the observed signal, expressed in terms of Gaussian sigma.

To obtain upper limits on the production cross section, we carried out a Monte Carlo simulation. In this way, the details of our peak search procedure, the peak shape, experimental resolution, corrections, background fluctuations, etc. were accounted for. The Monte Carlo simulated the presence of a peak, whose cross-section $\sigma_{M C}$ was varied from 0 to $20 \mathrm{nb} / \mathrm{sr}$. The purpose was to find the correspondence between the Monte Carlo peak strength and the probability to find a certain $\chi^{2}$ improvement, $\Delta \chi^{2}\left(\sigma_{M C}\right)$, due to such a peak when applying the search algorithm described above to the Monte Carlo spectra. We define the quantity $\Delta \chi_{90}^{2}\left(\sigma_{M C}\right)$ such that a Monte Carlo peak of crosssection $\sigma_{M C}$ results in a $\chi^{2}$ improvement greater than $\Delta \chi_{90}^{2}$ with $90 \%$ probability. Thus, if a value $\Delta \chi^{2}=\Delta \chi_{90}^{2}\left(\sigma_{M C}\right)$ is actually observed in the experiment, then we know that 
TABLE 1. Parameters of the largest observed bumps in each reaction channel. The range given for $\sigma_{\max }$ corresponds to the range $\Gamma=1-8 \mathrm{MeV}$ of assumed widths used in the peak search.

\begin{tabular}{ccccc}
\hline Channel & $\begin{array}{c}\text { Mass value of } \\
\text { largest peak } \\
(\mathrm{MeV})\end{array}$ & Significance & $\begin{array}{c}\text { Yield ratio } \\
\text { to } \Lambda(1520)\end{array}$ & $\begin{array}{c}\text { Cross section } \\
\text { limit } \sigma_{\text {max }} \\
(\mathrm{nb} / \mathrm{sr})\end{array}$ \\
\hline$e p \rightarrow e^{\prime} K^{+} X\left(\Sigma_{5}^{0}\right)$ & 1720 & $2.3 \sigma$ & $3.7 \%$ & $8-16$ \\
$e p \rightarrow e^{\prime} \pi^{+} X\left(N_{5}^{0}\right)$ & 1855 & $2.5 \sigma$ & $2.0 \%$ & $4-9$ \\
$e p \rightarrow e^{\prime} K^{-} X\left(\Theta^{++}\right)$ & 1570 & $3 \sigma$ & $1.4 \%$ & $3-6$ \\
\hline
\end{tabular}

we may have missed a real peak of cross section $\sigma \leq \sigma_{M C}$ with only $10 \%$ probability. Hence, we can take the Monte Carlo cross section $\sigma_{M C}$ for which $\Delta \chi_{90}^{2}\left(\sigma_{M C}\right)=\Delta \chi_{\text {exp }}^{2}$ as the $90 \%$ CL upper limit on the production cross section. The resulting values for our searches are given in Table 1.

\section{RESULTS AND CONCLUSIONS}

Figure 2 depicts the missing mass spectra obtained for the three reaction channels along with a fit to the most significant candidate peak found in each scan. The parameters of these peaks are given in Table 1 . These results were obtained with an assumed width of $\Gamma=5 \mathrm{MeV}$ for the Breit-Wigner function; other assumed widths yield very similar results, except that $\sigma_{\max }$ generally increases with increasing peak width, as expected. The table also gives the cross-section ratio of the respective largest peak to the cross section of the $\Lambda(1520)$ as measured in our experiment in the $K^{+}$channel.

There are several known or suspected resonances in our mass region [4], in particular several 3 or 4 -star $\Lambda$ and $\Sigma$ states in the ep $\rightarrow e^{\prime} K^{+} X$ channel. Most of these states are either wide $(>50 \mathrm{MeV})$ or have only been seen in partial-wave analyses. Taken together, they likely add up to a relatively smooth background. Still, we do see several broad structures in this channel, possibly the $\Sigma(1670)$ and $\Sigma(1775)$.

In conclusion, we do not observe statistically significant narrow $(\Gamma<10 \mathrm{MeV})$ structures in any of our three reaction channels. Any signals seen are consistent with background. Our experiment had a relatively small angular coverage, moderate statistics, and was kinematically incomplete, thus our ability to rule out pentaquark partner states or other narrow resonances in our search regions is naturally somewhat restricted.

\section{REFERENCES}

1. see, for example, S. Stepanyan et al. [CLAS collaboration], Phys. Rev. Lett. 91, 252001 (2003).

2. D. Diakonov, V. Petrov, and M. Polyakov, Z. Phys. A 359, 305 (1997).

3. S. Capstick et al., Phys. Lett. B 570, 185 (2003).

4. Review of Particle Physics, S. Eidelman et al., Phys. Lett. B 592, 1 (2004). 
(a)

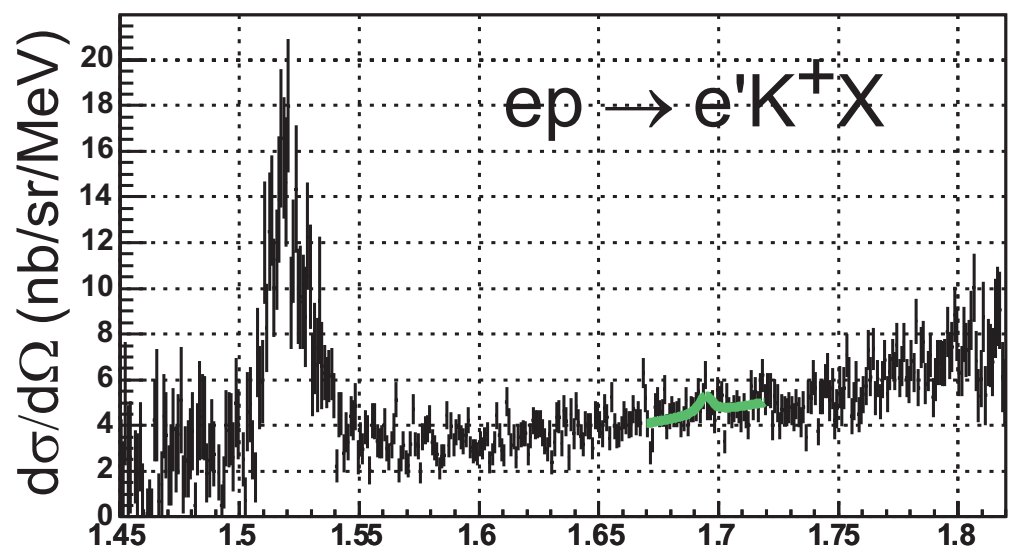

(b)

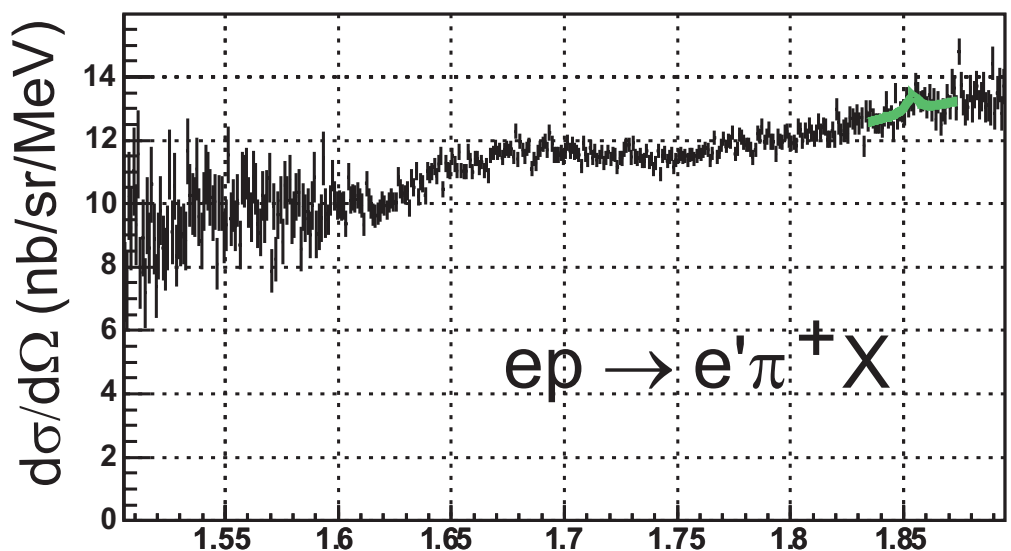

(c)

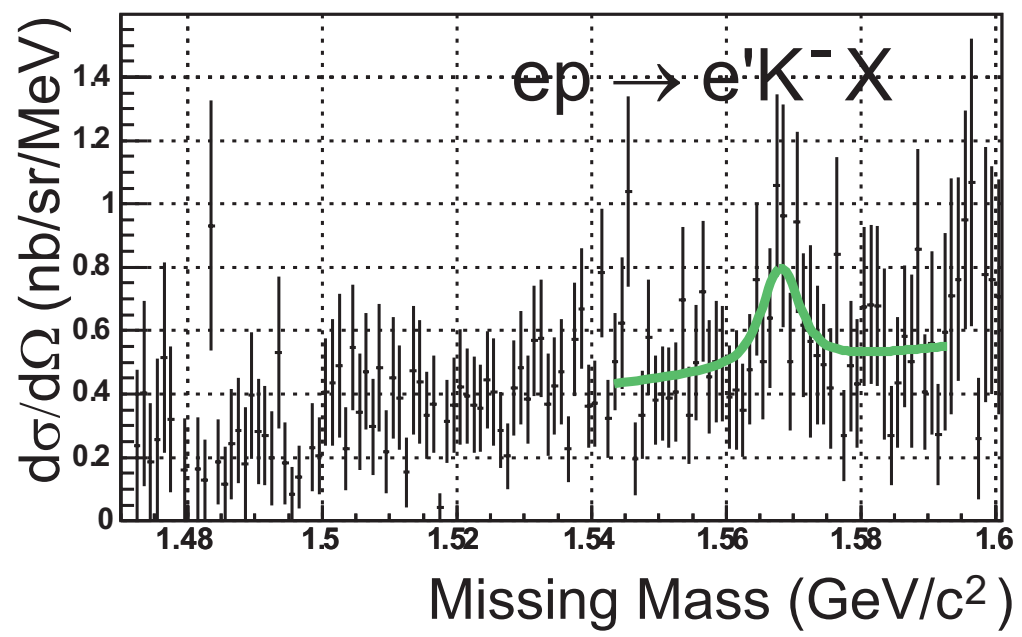

FIGURE 2. Final missing mass spectra obtained for the three reaction channels (a) $e p \rightarrow e^{\prime} K^{+} X$ ( $\Sigma_{5}^{0}$ search), (b) $e p \rightarrow e^{\prime} \pi^{+} X$ ( $N_{5}^{0}$ search), and (c) $e p \rightarrow e^{\prime} K^{-} X$ ( $\Theta^{++}$search). The fits to the most significant peak are shown in each spectrum; the parameters of these peaks are listed in Table 1. A peak width of $\Gamma=5 \mathrm{MeV}$ was assumed for the fits shown; other assumed widths yield similar results. The large peak in the $K^{+}$channel (a) is the $\Lambda(1520)$. 